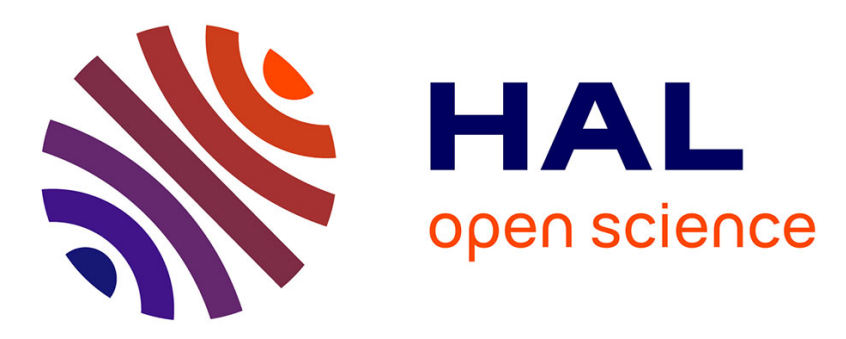

\title{
Numerical study of turbulent cavitating flows in thermal regime
}

Eric Goncalves da Silva, Dia Zeidan

\section{To cite this version:}

Eric Goncalves da Silva, Dia Zeidan. Numerical study of turbulent cavitating flows in thermal regime. International Journal of Numerical Methods for Heat and Fluid Flow, 2017, 27 (7), pp.1487-1503. 10.1108/HFF-05-2016-0202 . hal-01578647

\section{HAL Id: hal-01578647 https://hal.science/hal-01578647}

Submitted on 29 Aug 2017

HAL is a multi-disciplinary open access archive for the deposit and dissemination of scientific research documents, whether they are published or not. The documents may come from teaching and research institutions in France or abroad, or from public or private research centers.
L'archive ouverte pluridisciplinaire HAL, est destinée au dépôt et à la diffusion de documents scientifiques de niveau recherche, publiés ou non, émanant des établissements d'enseignement et de recherche français ou étrangers, des laboratoires publics ou privés. 


\section{Numerical study of turbulent cavitating flows in thermal regime Eric Goncalves ${ }^{a}$ and Dia Zeidan ${ }^{b}$ \\ ${ }^{a}$ ISAE-ENSMA, Institut Pprime, UPR 3346 CNRS, Poitiers, France. \\ ${ }^{b}$ School of Basic Sciences and Humanities, German Jordanian University, Amman, Jordan.}

\section{Abstract}

Purpose The aim of this work is to quantify the relative importance of the turbulence modelling for cavitating flows in thermal regime. A comparison of various transportequation turbulence models and a study of the influence of the turbulent Prandtl number appearing in the formulation of the turbulent heat flux are proposed. Numerical simulations are performed on a cavitating Venturi flow for which the running fluid is freon R-114 and results are compared with experimental data.

Design/methodology/approach A compressible, two-phase, one-fluid Navier-Stokes solver has been developed to investigate the behaviour of cavitation models including thermodynamic effects. The code is composed of three conservation laws for mixture variables (mass, momentum and total energy) and a supplementary transport equation for the volume fraction of gas. The mass transfer between phases is closed assuming its proportionality to the mixture velocity divergence.

Findings The influence of turbulence model as regard to the cooling effect due to the vaporization is weak. Only the $k-\varepsilon$ Jones-Launder model under-estimates the temperature drop. The amplitude of the wall temperature drop near the Venturi throat increases with the augmentation of the turbulent Prandtl number.

Originality The interaction between RANS turbulence closure and non isothermal phase transition is rarely studied. It is the first time such a study on the turbulent Prandtl number effect is reported in cavitating flows.

Keywords cavitation; thermodynamic effects; homogeneous model; RANS simulation; turbulence models; turbulent Prandtl number 


\section{Introduction}

The turbopump design stages of liquid rocket engines requires an accurate prediction of the performance of cavitating cryogenic inducers involving thermodynamic effects. For cryogenic fluids, the liquid-vapour density ratio is lower than that of typical fluids (cold water) and consequently more liquid mass has to vaporize to sustain a cavity. Therefore evaporative cooling effects are more pronounced and the temperature of the liquid in the immediate vicinity of the liquid-vapour interface is depressed below the free-stream temperature. The temperature depression, negligible in cold water, is quite substantial. The local cooling effect delays the cavitation phenomenon and reduces the local vapour pressure of the fluid, which leads to a lower observed cavity pressure. Typically, this results in an improved mean performance of cryogenic pumps. Early studies about thermal effects were generally focused on obtaining correlations for temperature depression as a function of flow conditions and liquid properties. Classical methods include the B-factor theory (Stahl et al., 1956) to characterize the sensitivity of fluids to thermodynamic effects. Another approach is based on the bubble dynamics model through the use of the Rayleigh-Plesset equation. This model is capable of handling either single bubbles or clouds of bubbles that grow and decrease through a pressure field (Fujikawa et al., 2080). Yet, when thermal effects are involved, the liquid inertia becomes rapidly negligible and the evolution is controlled by the heat flux provided by the liquid at the bubble surface (Florschuetz and Chao, 1965, Prosperetti and Plesset, 1978).

A considerable literature exists on the simulations of two-phase fluid flow problems. See for example (Voller, 2016, Wang et al., 2015, Liang et al., 2014, Paniagua et al., 2013, Daude and Galon, 2016, Zeidan, 2016, Zeidan et al., 2014) and references therein. These simulations are mainly based on the widely used two-fluid model which is derived on the basis of averaging values for all the flow parameters and state variables ranging from seven to three equations. For instance, a seven-equation two-phase model has been proposed

in, e.g. see (Saurel and Metayer, 2001) with velocity and pressure non-equilibrium where interfacial forces appear between phases developing momentum and energy exchange. 
Another reduced five-equation model has been derived from a two-fluid model with the assumptions of velocity and pressure equilibrium (Kapila et al., 2001). It is composed of four conservation laws: two for masses, one for the mixture momentum and one for the mixture energy. It is completed by an equation for a non-conservative quantity describing the flow topology, usually the void ratio. Such a model has been used for inviscid high speed cavitating applications (Saurel et al., 2008, Rodio and Abgrall, 2015). By assuming the thermal equilibrium between phases, a four-equation model can be obtained. A very popular formulation, originally developed to simulate turbulent cavitating flows in cold water, has been adapted to cryogenic applications (Utturkar et al., 2005, Tseng and Shyy, 2010, Huang et al., 2014). It is composed by three conservation laws for mixture quantities (mass, momentum, energy) plus a mass equation for the vapour or liquid density including a cavitation source (different sets of models are presented in (Utturkar et al., 2005)). Yet, this family of models are not thermodynamically well-posed and does not respect thermodynamic constraints such as mixture speed of sound conditions (Goncalves and Patella, 2011). With the assumption of complete thermodynamic equilibrium between phases (local temperature, pressure and free Gibbs enthalpy equality between phases), we obtain the 3-equation models or Homogeneous Equilibrium Models (HEM). An equation of state (EOS) is necessary to close the system. Different closure relations (tabulated EOS or combination of pure phase EOSs) that link the pressure to the thermodynamic variables have been proposed (Cooper, 1967, Clerc, 2000, Goncalves and Patella, 2010). The objective of this paper is to evaluate the sensitivity related to the choice of turbulence models on the cavitation prediction for liquid rocket engines. A canonical cavitating flow on a Venturi geometry is chosen for which the running fluid is freon R-114 $\left(\mathrm{C}_{2} \mathrm{Cl}_{2} \mathrm{~F}_{4}\right)$. The cavitation model is based on a transport-equation for the void fraction in which the mass transfer between phases appear explicitly. This term is closed by assuming its proportionality with the velocity divergence (Goncalves, 2013, Goncalves and Charriere, 2014, Goncalves and Zeidan, 2016a,b). We investigate the thermal effects as regard to the temperature drop inside the cavitation pocket by testing the influence of (i) different transport-equation turbulence models associated with the Boussinesq assumption, (ii) 
the turbulent heat flux through a turbulent Fourier law. The numerical simulations are compared with experimental data based on void ratio profiles and wall temperature depression. This paper is organized as follows. In Section 2, we present the cavitation model, the mass transfer closure relations and the mixture equation of states. Section 3 describes the Reynolds-Averaged Navier-Stokes formulation (RANS) with the turbulence modeling and the numerical schemes for the solving the model equations. A description of the Venturi case is given in Section 4. This is followed by simulation results demonstrating the influence of turbulence model. The last section is a conclusion.

\section{Cavitation model}

The homogeneous mixture approach is used to model two-phase flows. In addition, the phases are assumed to be in mechanical equilibrium: they share the same pressure $P$. Although the temperature-equalizing time is larger than the pressure and velocity relaxation times, as noted in (Kapila et al., 2001), it is possible to consider a single-temperature model as an approximate model of flow if the difference of phase temperatures is not too big.

\subsection{A four-equation single-temperature model}

The model consists of three conservation laws for mixture quantities and an additional equation for the void ratio (Goncalves, 2013). We present below the inviscid one-dimensional 
equations, expressed with the vector of variables $w=(\rho, \rho u, \rho E, \alpha)$ :

$$
\begin{array}{rll}
\frac{\partial \rho}{\partial t}+\frac{\partial \rho u}{\partial x} & = & 0 \\
\frac{\partial(\rho u)}{\partial t}+\frac{\partial\left(\rho u^{2}+P\right)}{\partial x} & = & 0 \\
\frac{\partial(\rho E)}{\partial t}+\frac{\partial(\rho u H)}{\partial x} & = & 0 \\
\frac{\partial \alpha}{\partial t}+u \frac{\partial \alpha}{\partial x} & = & K \frac{\partial u}{\partial x}+\frac{\dot{m}}{\rho_{I}} \\
K=\frac{\rho_{l} c_{l}^{2}-\rho_{v} c_{v}^{2}}{\frac{\rho_{l} c_{l}^{2}}{1-\alpha}+\frac{\rho_{v} c_{v}^{2}}{\alpha}} & \text { and } \quad \frac{1}{\rho_{I}}=\frac{\frac{c_{v}^{2}}{\alpha}+\frac{c_{l}^{2}}{1-\alpha}}{\frac{\rho_{l} c_{l}^{2}}{1-\alpha}+\frac{\rho_{v} c_{v}^{2}}{\alpha}}
\end{array}
$$

where $E=e+u^{2} / 2$ and $H=h+u^{2} / 2$ denote the mixture total energy and the mixture total enthalpy, respectively. The quantity $K$ reflects the effects of changes in volume of each phase, $\rho_{I}$ is the interfacial density, $\dot{m}$ is the mass transfer between phases and $c_{k}$ the speed of sound of the phase $k$.

\subsection{Pure phase EOS}

The liquid density $\rho_{l}$ is assumed to be in its equilibrium state at the reference temperature: $\rho_{l}=\rho_{l}^{\text {sat }}\left(T_{r e f}\right)$. The vapour density $\rho_{v}$ follows the stiffened gas EOS and varies with the temperature. The convex stiffened gas EOS relations are (see (Metayer et al., 2004)):

$$
\begin{aligned}
P(\rho, e) & =(\gamma-1) \rho(e-q)-\gamma P_{\infty} \\
P(\rho, T) & =\rho(\gamma-1) C_{v} T-P_{\infty} \\
T(\rho, h) & =\frac{h-q}{C_{p}}
\end{aligned}
$$

where $\gamma=C_{p} / C_{v}$ is the heat capacity ratio, $C_{p}$ and $C_{v}$ are thermal capacities, $q$ the energy of formation and $P_{\infty}$ is a constant reference pressure. The speed of sound $c$ is given by:

$$
c^{2}=\gamma \frac{P+P_{\infty}}{\rho}=(\gamma-1) C_{p} T
$$

The stiffened gas parameters for freon R-114 are given in Table 1. 


\begin{tabular}{lllllll}
\hline & $T_{\text {ref }}(\mathrm{K})$ & $\gamma$ & $P_{\infty}(\mathrm{Pa})$ & $\mathrm{q}(\mathrm{J} / \mathrm{kg})$ & $C_{p}(\mathrm{~J} / \mathrm{K} . \mathrm{kg})$ & $\rho^{\text {sat }}\left(\mathrm{kg} / \mathrm{m}^{3}\right)$ \\
\hline liquid & 293 & 1.1 & $3.835 \times 10^{7}$ & $-6.910 \times 10^{4}$ & 984 & 1470.3 \\
vapour & 293 & 1.07 & 0 & $1.424 \times 10^{5}$ & 700 & 13.52 \\
\hline
\end{tabular}

Table 1: Parameters of the stiffened gas EOS for freon R-114 at saturation.

\subsection{Closure relation for the mass transfer}

By assuming that the mass transfer is proportional to the divergence of the velocity, it is possible to build a family of models (Goncalves, 2013, Goncalves and Charriere, 2014) in which the mass transfer is expressed as:

$$
\dot{m}=\frac{\rho_{l} \rho_{v}}{\rho_{l}-\rho_{v}}\left(1-\frac{c^{2}}{c_{\text {wallis }}^{2}}\right) \frac{\partial u}{\partial x}
$$

where $c_{\text {wallis }}$ is the propagation velocity of acoustic waves without mass transfer (Wallis, 1967). This speed of sound is expressed as a weighted harmonic mean of speeds of sound of each phase:

$$
\frac{1}{\rho c_{\text {wallis }}^{2}}=\frac{\alpha}{\rho_{v} c_{v}^{2}}+\frac{1-\alpha}{\rho_{l} c_{l}^{2}}
$$

\subsection{Mixture EOS}

To close the system and to compute the mixture pressure and the mixture temperature, a equation of state for the mixture is necessary. A sinusoidal relation can be considered for the mixture (Goncalves and Patella, 2010). When the pressure is smaller than $P_{\text {vap }}(T)+$ $\Delta P$, the following relationship applies:

$$
P(\alpha, T)=P_{v a p}(T)+\left(\frac{\rho_{l}^{s a t}-\rho_{v}^{s a t}}{2}\right) c_{0}^{2} \sin ^{-1}(1-2 \alpha)
$$

This EOS introduces a small non-equilibrium effect on the pressure quantified by the quantity $\Delta P$. For a void ratio value of 0.5 , the pressure is equal to the saturation pressure $P_{\text {vap }}(T)$ at the local temperature $T$. The saturation values $\rho_{l}^{\text {sat }}$ and $\rho_{v}^{\text {sat }}$ are evaluated at the reference temperature $T_{r e f}$. The quantity $c_{0}$, which has the dimension of a velocity, 
is a parameter of the model. The pressure continuity between the liquid and the mixture is given by:

$$
\frac{\pi}{2} \frac{\rho_{l}^{s a t}-\rho_{v}^{s a t}}{2} c_{0}^{2}=\rho_{l}^{s a t}\left(\gamma_{l}-1\right) C_{v_{l}} T_{\text {ref }}-P_{\infty}^{l}-P_{\text {vap }}\left(T_{\text {ref }}\right)
$$

This relation determines $c_{0}$ for given values of saturation conditions. In the present study, $c_{0}=0.74 \mathrm{~m} / \mathrm{s}$.

We assume that the vaporization pressure varies linearly with the temperature:

$$
P_{\text {vap }}(T)=P_{\text {vap }}\left(T_{r e f}\right)+\frac{d P_{v a p}}{d T}\left(T-T_{\text {ref }}\right)
$$

The constant quantity $d P_{\text {vap }} / d T$ is evaluated using a thermodynamic table around a reference temperature. In the present study, $d P_{\text {vap }} / d T=5900 \mathrm{~Pa} / \mathrm{K}$.

The speed of sound in the mixture can be written as (Goncalves and Patella, 2010):

$$
\begin{aligned}
c^{2} & =\frac{\frac{\rho_{v} \rho_{l}}{\rho\left(\rho_{l}-\rho_{v}\right)}\left(h_{v}-h_{l}\right) \frac{d P_{v a p}}{d T}+\rho C_{p}(Y) c_{T}^{2}}{\rho C_{p}(Y)-\frac{d P_{v a p}}{d T}} \\
C_{p}(Y) & =Y C_{p_{v}}+(1-Y) C_{p_{l}} \\
c_{T}^{2} & =\left(\frac{\partial P}{\partial \rho}\right)_{s}=\left(\frac{\partial P}{\partial \rho}\right)_{T}=\frac{c_{0}^{2}}{2 \sqrt{\alpha(1-\alpha)}}
\end{aligned}
$$

Where $c_{T}$ is the isothermal speed of sound (i.e. when $d P_{\text {vap }} / d T=0$ ) and $Y=\alpha \rho_{v} / \rho$ is the vapour mass fraction. The four-equation system is hyperbolic with eigenvalues: $\lambda_{1}=u-c, \lambda_{2,3}=u$ and $\lambda_{4}=u+c$.

Properties of the EOS as regard to both convexity and mixture speed of sound conditions have been studied in (Goncalves and Patella, 2010).

\section{Reynolds averaged Navier-Stokes equations}

For turbulent computations, the compressible one-fluid RANS equations are used, coupled with a one- or two-equation turbulence model. These equations can be expressed as:

$$
\frac{\partial w}{\partial t}+\operatorname{div}\left(F_{c}-F_{v}\right)=S
$$




$$
w=\left(\begin{array}{c}
\rho \\
\rho \vec{V} \\
\rho E \\
\alpha \\
\rho k \\
\rho \Psi
\end{array}\right) ; \quad F_{c}=\left(\begin{array}{c}
\rho \vec{V} \\
\rho \vec{V} \otimes \vec{V}+p \overline{\bar{I}} \\
(\rho E+p) \vec{V} \\
\alpha \vec{V} \\
\rho k \vec{V} \\
\rho \Psi \vec{V}
\end{array}\right) \quad ; \quad F_{v}=\left(\begin{array}{c}
0 \\
\overline{\overline{\tau^{v}}}+\overline{\overline{\tau^{t}}} \\
\left(\overline{\overline{\tau^{v}}}+\overline{\overline{\tau^{t}}}\right) \cdot \vec{V}-Q^{v}-Q^{t} \\
0 \\
\left(\mu+\mu_{t} / \sigma_{k}\right) \operatorname{grad} k \\
\left(\mu+\mu_{t} / \sigma_{\Psi}\right) \operatorname{grad} \Psi
\end{array}\right)
$$

where $w$ denotes the conservative variables and the void ratio, $F_{c}$ and $F_{v}$ the convective and viscous flux densities and $S$ the source terms, which concern the void ratio equation and the turbulent transport equation. $k$ is the mixture turbulent kinetic energy and $\Psi$ is a turbulent variable. The exact expression of the eddy-viscosity $\mu_{t}$ and the source terms depend on the turbulence model, as well as the constants $\sigma_{k}$ and $\sigma_{\Psi}$.

The total stress tensor $\overline{\bar{\tau}}$ is evaluated using the Stokes hypothesis, Newton's law and the Boussinesq assumption. The total heat flux vector $Q$ is obtained from the Fourier law involving a turbulent thermal conductivity $\lambda_{t}$.

$$
\begin{aligned}
& \overline{\bar{\tau}}=\overline{\overline{\tau^{v}}}+\overline{\overline{\tau^{t}}}=\left(\mu+\mu_{t}\right)\left[\left(\operatorname{grad} \vec{V}+(\operatorname{grad} \vec{V})^{t}\right)-\frac{2}{3}(\operatorname{div} \vec{V}) \overline{\bar{I}}\right]+\frac{2}{3} \rho k \overline{\bar{I}} \\
& Q=Q^{v}+Q^{t}=-\left(\lambda+\lambda_{t}\right) \operatorname{grad} T
\end{aligned}
$$

In the pure liquid, the viscosity is determined by an exponential law and, in pure vapour, the viscosity follows the Sutherland law. The mixture viscosity is defined as the arithmetic mean of the liquid and vapour viscosities:

$$
\begin{aligned}
\mu_{l}(T) & =\mu_{0_{l}} \exp (B / T) \\
\mu_{v}(T) & =\mu_{0_{v}} \sqrt{\frac{T}{293}} \frac{1+T_{S} / 293}{1+T_{S} / T} \\
\mu(T, \alpha) & =\alpha \mu_{v}(T)+(1-\alpha) \mu_{l}(T)
\end{aligned}
$$

where $\mu_{0_{l}}, \mu_{0_{v}}, B$ and $T_{S}$ are constant parameters.

The mixture thermal conductivity $\lambda$ is also defined as the arithmetic mean of the 
liquid and vapour values:

$$
\lambda(\alpha, T)=\alpha \frac{\mu_{v}(T) C_{p_{v}}}{P_{r_{v}}}+(1-\alpha) \frac{\mu_{l}(T) C_{p_{l}}}{P_{r_{l}}}
$$

\subsection{Wall modelling}

For the modelling of flow close to the wall, a two-layer wall law approach is used (Goncalves and Houdeville, 2001):

$$
\begin{aligned}
& u^{+}=y^{+} \quad \text { if } y^{+}<11.13 \\
& u^{+}=\frac{1}{\kappa} \ln y^{+}+5.25 \text { if } y^{+}>11.13 \\
& u^{+}=\frac{u}{U_{\tau}} \quad ; \quad y^{+}=\frac{y U_{\tau}}{\nu_{w}} \quad ; \quad U_{\tau}^{2}=\frac{\tau_{w}}{\rho_{w}}
\end{aligned}
$$

where $\kappa=0.41$ is the von Karman constant and the subscript 'w' is used for a wall value. We assume that wall functions are similar in a two-phase flow and in a single-phase flow. This assumption have been studied in (Goncalves and Decaix, 2012) and comparisons were proposed with a thin boundary layer approach.

At the wall, an adiabatic condition is imposed. Gradients of temperature and pressure are assumed to be null. For the void ratio wall value, we use the following formulation:

$$
\alpha_{w}=\frac{\rho_{w}-\rho_{l}^{\text {sat }}\left(T_{r e f}\right)}{\rho_{v}\left(T_{w}\right)-\rho_{l}^{\text {sat }}\left(T_{r e f}\right)} \quad \text { with } \quad \rho_{v}\left(T_{w}\right)=\frac{P_{w}}{C_{v_{v}}\left(\gamma_{v}-1\right) T_{w}}
$$

\subsection{Turbulence models}

A comparison with various transport-equation turbulence models is proposed. We consider five models: the one-equation Spalart-Allmaras model (SA) (Spalart and Allmaras, 1994), the Jones-Launder $k-\varepsilon$ model (KE) (Jones and Launder, 1972), the Smith $k-\ell$ model (KL) (Smith, 1994), the Wilcox $k-\omega$ model (KO) (Wilcox, 1988) and the Menter SST $k-\omega$ model (SST) (Menter, 1994). 


\subsection{Turbulent heat flux}

We introduce a constant turbulent Prandtl number hypothesis. The mixture turbulent thermal conductivity is given by the arithmetic mean of the liquid and vapour values:

$$
\lambda_{t}=\alpha \lambda_{v}^{t}+(1-\alpha) \lambda_{l}^{t}=\alpha \frac{\mu_{t} C_{p_{v}}}{P_{r_{t}}}+(1-\alpha) \frac{\mu_{t} C_{p_{l}}}{P_{r_{t}}} \simeq \frac{\mu_{t} C_{p}(Y)}{P_{r_{t}}}
$$

where the turbulent Prandtl number $P_{r_{t}}$ is usually set to 1 , that is to say the momentum and thermal eddy diffusivities are in the same order of magnitude. For two-dimensional boundary layer, this number is evaluated by

$$
P_{r_{t}}=\frac{\nu_{t}}{\alpha_{t}}=\frac{\overline{u^{\prime} v^{\prime}} \frac{\partial T}{\partial y}}{\overline{v^{\prime} T^{\prime}} \frac{\partial u}{\partial y}}
$$

A large quantity of experimental, analytical and numerical works have been devoted to the evaluation of this number in the near-wall region of single-phase boundary layer (see review by (Kays, 1994)). A clear lack of agreement appears in the literature. For a twophase boundary layer involving phase transition, the question is totally open. We propose to test different values of $P_{r_{t}}$ from 0.25 to 1.5 .

\subsection{Numerical methods}

The numerical simulations are carried out using an implicit CFD code based on a finitevolume discretization. For the mean flow, the convective flux density vector on a cell face is computed with the Jameson-Schmidt-Turkel scheme (Jameson et al., 1981). The artificial viscosity includes a second-order dissipation term $D_{2}$ and a fourth-order dissipation term $D_{4}$, which involve two tunable parameters. The viscous terms are discretized by a secondorder space-centered scheme. For the turbulence transport equations, the upwind Roe scheme (Roe, 1981) is used to obtain a more robust method. The second-order accuracy is obtained by introducing a flux-limited dissipation. The numerical treatment of boundary conditions is based on the use of the characteristic relationships. More details are given in (Goncalves, 2013). 


\section{Application: the Venturi geometry}

\subsection{Experimental conditions}

The freon R-114 experimental facility of the CREMHyG is a closed loop operating with a reference pressure, obtained by pressurizing a tank with nitrogen gas. The loop is fitted with a test section having the shape of a two-dimensional Venturi, characterized by a convergence angle of $4.3^{\circ}$ and a divergence angle of $4^{\circ}$ (Fig. 1). The edge forming the throat of the Venturi is used to fix the separation point of the cavitation cavity. This geometry is equipped with three probing holes to take various measurements. Optical probes and micro-thermocouples are used to evaluate the local void ratio and the wall temperature, respectively. For this geometry, the selected operation point is characterized by the following physical parameters (Fruman et al., 1999):

- the inlet velocity $V_{\text {inlet }}=14.4 \mathrm{~m} / \mathrm{s}$;

- the reference temperature $T_{\text {ref }}=293 \mathrm{~K}$;

- the reference length $L_{r e f}=0.252 \mathrm{~mm}$, corresponding to the chord of a blade turbomachinery;

- the Reynolds number $R e_{L_{r e f}}=\frac{V_{\text {inlet }} \times L_{r e f}}{\nu\left(T_{r e f}\right)}=18.4 \times 10^{6}$;

- the cavitation parameter in the inlet section $\sigma_{\text {inlet }}=\frac{P_{\text {inlet }}-P_{\text {vap }}\left(T_{\text {ref }}\right)}{0.5 \rho V_{\text {inlet }}^{2}} \simeq 0.55$.

With these parameters, a cavity length $L_{\text {cav }}$ around $80 \mathrm{~mm}$ was obtained, with a relatively stable aspect.

A simple heat balance between the two phases can estimate the scale of temperature difference $\Delta T^{*}$ caused by thermal effects.

$$
\Delta T^{*}=\frac{\rho_{v} L_{v a p}}{\rho_{l} C_{p_{l}}}
$$

where $L_{v a p}$ is the latent heat. Values of this temperature difference are given in Table 2 for water, freon R-114 and liquid hydrogen $\mathrm{LH}_{2}$ in cryogenic conditions. Freon R-114 provides a similar $\Delta T^{*}$ at room temperature in comparison with $L H_{2}$ and is used as a substitute fluid for turbopump applications. 


\begin{tabular}{lllllll}
\hline fluid & $T_{\text {ref }}(\mathrm{K})$ & $\rho_{L}\left(\mathrm{~kg} / \mathrm{m}^{3}\right)$ & $\rho_{V}\left(\mathrm{~kg} / \mathrm{m}^{3}\right)$ & $P_{\text {vap }}(\mathrm{Pa})$ & $\frac{d P_{\text {vap }}}{d T}(\mathrm{~Pa} / \mathrm{K})$ & $\Delta T^{*}(\mathrm{~K})$ \\
\hline water & 293 & 998 & 0.02 & 2339 & 143 & 0.01 \\
R-114 & 293 & 1470.3 & 13.52 & 181100 & 5900 & 1.22 \\
LH2 & 21 & 70.0 & 1.62 & 124720 & 35300 & 1.01 \\
LH2 & 21 & 67.4 & 2.60 & 209320 & 50000 & 1.41 \\
\hline
\end{tabular}

Table 2: Thermodynamic properties of different fluids at saturation.

\subsection{Computational parameters and mesh}

The grid is a H-type topology. The mesh contains 251 nodes in the flow direction and 77 in the orthogonal direction. A special contraction is applied in the main flow direction just after the throat to better simulate the two-phase flow area (see Fig. 2). $y+$ values in first adjacent cells to walls vary between 35 to 50 for a non cavitating simulation.

All cavitating simulations are steady computations, which are started from the non cavitating numerical solution. Computations have been performed with a CFL number equal to 0.5 and dissipation parameters equal to 1 and 0.045 .

\subsection{Influence of the turbulence model}

Different calculations were performed in order to obtain a cavitation sheet with a length close to $80 \mathrm{~mm}$. Comparisons between different cavitation models have been presented in (Goncalves and Charriere, 2014) using the Spalart-Allmaras turbulence model. In the following, we study the turbulence model influence.

A qualitative view of the cavitation pocket attached to the throat is presented in Figure 3 with the void ratio field (top) and the temperature deficit $T-T_{\text {ref }}$ (K) (down). Results have been obtained using the $k-\omega$ SST Menter and the $k-\varepsilon$ Jones-Launder models. The cooling effect due to the vaporization process is clearly observed (negative values). In the recompression area, a warming effect is exhibited as observed previously 
in (Goncalves, 2014) using the Spalart-Allmaras model. Locally, the temperature exceeds the freestream temperature and values reach more than $5 \mathrm{~K}$. It is certainly due to the collapse of bubbles which causes the rapid recuperation of the temperature, as related by experimental measurements using infra-red thermography (Petkovsek and Dular, 2013). Such a phenomenon is predicted by all simulations with the five considered turbulence models. The qualitative view is quite similar for all simulations.

Complementary analyses concern local void ratio profiles inside the cavity and the wall temperature depression. The experimental void ratio profiles are obtained with a post processing algorithm from the signal of the optical probe (Fruman et al., 1999). Figure 4 (on the left) illustrates the numerical void ratio obtained with the five turbulence models in comparison with the measurements at stations 1 to 3 . We clearly observe that numerical results are quite superimposed. As regard to the wall value of the void ratio, a large discrepancy appears: the numerical wall values are largely over-predicted in comparison with the experimental data at stations 2 and 3. Moreover, the decrease of the void ratio close to the wall is absolutely not reproduced. This behaviour is probably due to the considered wall condition for the void ratio (equation 25).

For the analysis of the flow, we added a numerical probe inside the cavity at abscissa $x=74 \mathrm{~mm}$ named station 5 . The temperature depression $T_{r e f}-T$ at stations 1,3 and 5 is plotted in Figure 4 (on the right) for the five turbulence models. The cooling effect is more pronounced at station 1, close to the Venturi throat. The shape of the profiles is quite similar for all simulations. Near the wall, small discrepancies are noticeable at stations 1 and 2 . The wall temperature depression at station 1 is about $2.2 \mathrm{~K}( \pm 0.2 \mathrm{~K})$ for the experimental data. Except the under-estimation provided by the Jones-Launder model (around $1.8 \mathrm{~K}$ ), results are in very good agreement with the measurement. At station 2, similarly the Jones-Launder simulation provides a lower temperature drop in comparison with other models. Finally, at station 5, in the end part of the cavitation pocket, all models provide similar profiles. We note that the $k-\varepsilon$ model also predicted the highest void ratio wall values at stations 1 to 3 . 
To investigate the effect of turbulence models, profiles of viscosity ratio $\mu_{t} / \mu$ (on the left) and thermal conductivity ratio $\lambda_{t} / \lambda$ (on the right) are plotted in Figure 5 at stations 1 and 3 for the five turbulence models. Globally, discrepancies appear on the thickness of profiles and on the maximum values. The Jones-Launder model provides the thinnest profiles and the lowest values. On the contrary, the Smith $k-\ell$ model gives the highest values but it is not associated to a larger temperature drop.

\subsection{Influence of the turbulent Prandtl number}

As regard to the previous study, the Spalart-Allmaras model is chosen for all simulations. Different values of the turbulent Prandtl number are tested from 0.25 to 1.5. With a small value of $P_{r_{t}}$, the thermal eddy diffusivity is faster than the momentum eddy diffusivity and a reduction of the thermal amplitude is expected.

First, a qualitative view of the cavitation pocket is illustrated in Figure 6 where the void ratio and temperature deficit fields are plotted. Three simulations are compared: $P_{r_{t}}=1.5$ (top), $P_{r_{t}}=1$ (middle) and $P_{r_{t}}=0.25$ (down). No clear discrepancies are put in evidence between simulations.

Secondly, the void ratio profiles at station 1 to 3 are presented in the left part of Figure 7. Using the smallest value $P_{r_{t}}=0.25$, the thickness of the cavity is a little reduced, especially at station 3 . For the highest value $P_{r_{t}}=1.5$, the wall value is more important (around $75 \%$ ) and the S-shape at station 3 is not predicted. For the intermediate values, profiles are quite similar.

Profiles of temperature depression $T_{r e f}-T(\mathrm{~K})$ at stations 1,3 and 5 are plotted in the

right part of Figure 7. At station 1, discrepancies are noticeable for the wall temperature depression. Higher is the turbulent Prandtl number, stronger is the temperature depression. Using the highest value $P_{r_{t}}=1.5$, the wall temperature difference reaches $2.5 \mathrm{~K}$. 
At stations 3 and 5, results are relatively similar. A small discrepancy appears on the maximum value at the interface of the cavitation pocket.

Complementary analyses are proposed for turbulent quantities. The viscosity ratio $\mu_{t} / \mu$ and the thermal conductivity ratio $\lambda_{t} / \lambda$ are plotted in Figure 8 for different values of $P_{r_{t}}$. Viscosity ratio profiles are quite similar. The conductivity ratio profiles show large difference. The hierarchy between the five values of $P_{r_{t}}$ is well illustrated. The maximum ratio obtained with the smallest value $P_{r_{t}}=0.25$ is around 4.7 times the value predicted for the case $P_{r_{t}}=1$. For the case $P_{r_{t}}=1.5$, the augmentation is around a factor 1.4. The conductivity ratio is not directly proportional to the viscosity ratio through the Prandtl numbers in two-phase flow.

Finally, the wall temperature depression $T_{r e f}-T(\mathrm{~K})$ is presented in Figure 9 along the Venturi divergent function of the dimensionless abscissa $x^{*}=\left(x-x_{\text {throat }}\right) / L_{\text {cav }}$. The experimental value corresponds to the wall measurement at station 1 . Close to the throat, for $x^{*} \leq 0.1$, we observe a peak of the temperature depression followed by a decrease along the wall up to the closure part of the cavity. As observed on the temperature profile at station 1 , this peak is more pronounced for the highest value $P_{r_{t}}=1.5$. From the abscissa $x^{*}=0.2$, all results are quite superimposed. At the closure part of cavities, discrepancies are noticeable due to the fact that the cavity length $L_{c a v}$ is not exactly the same for all simulations. Negative values of the quantity $T_{r e f}-T$ illustrate the warming effect in the recompression area.

\section{Conclusion}

One-fluid single-temperature RANS simulations were performed to study cavitation pockets developing along a Venturi geometry in which the working fluid is freon R-114. An investigation of the influence of various transport-equation turbulence models and the turbulent Prandtl number was led. Numerical results have been compared with experimental data, especially as regard to the cooling effect due to the vaporization process. 
These investigations showed globally the weak effect of the turbulence model, only the $k-\varepsilon$ model provided a smaller temperature depression in comparison with other models. It is difficult to explain this behaviour. The physical mechanisms involved are not clear and the interaction between heat and mass transfers in a turbulent boundary layer remain challenging to model. Similarly, the influence of the turbulent Prandtl number is rather small. The amplitude of the wall temperature drop is modified near the throat and increases with the augmentation of $P_{r_{t}}$.

Additional works are in progress to pursue comparative analyses and to develop 2-temperature models. Moreover, further experimental cavitating works concerning local measurements in thermosensitive fluid are capital to allow model calibration and validation. 


\section{References}

Clerc, S. (2000), 'Numerical simulation of the homogeneous equilibrium model for twophase flows', Journal of Computational Physics 161(1), 354-375.

Cooper, P. (1967), 'Analysis of single and two-phase flow in turbopump inducers', Journal of Fluids Engineering 89, 577-588.

Daude, F. and Galon, P. (2016), 'On the computation of the Baer-Nunziato model using ALE formulation with HLL- and HLLC-type solvers towards fluidstructure interactions', Journal of Computational Physics 304, 189-230.

Florschuetz, L. and Chao, B. (1965), 'On the mechanics of vapor bubble collapse', Journal of Heat Transfer 87, 209-220.

Fruman, D., Reboud, J. and Stutz, B. (1999), 'Estimation of thermal effects in cavitation of thermosensible liquids', Int. Journal of Heat and Mass Transfer (42), 3195-3204.

Fujikawa, S., Okuda, M. and Akamatsu, T. (2080), 'Non-equilibrium vapour condensation on a shock-tube endwall behind a reflected shock wave', Journal of Fluid Mechanics (183), 293-324.

Goncalves, E. (2013), 'Numerical study of expansion tube problems: Toward the simulation of cavitation', Computers \& Fluids 72, 1-19.

Goncalves, E. (2014), 'Modeling for non isothermal cavitation using 4-equation models', International Journal of Heat and Mass Transfer 76, 247-262.

Goncalves, E. and Charriere, B. (2014), 'Modelling for isothermal cavitation with a fourequation model', International Journal of Multiphase Flow 59, 54-72.

Goncalves, E. and Decaix, J. (2012), 'Wall model and mesh influence study for partial cavities', European Journal of Mechanics B/Fluids 31(1), 12-29. 
Goncalves, E. and Houdeville, R. (2001), 'Reassessment of the wall functions approach for RANS computations', Aerospace Science and Technology 5, 1-14.

Goncalves, E. and Patella, R. F. (2010), 'Numerical study of cavitating flows with thermodynamic effect', Computers \& Fluids 39(1), 99-113.

Goncalves, E. and Patella, R. F. (2011), 'Constraints on equation of state for cavitating flows with thermodynamic effects', Applied Math. and Computation 217, 5095-5102.

Goncalves, E. and Zeidan, D. (2016a), 'Numerical simulation of unsteady cavitation in liquid hydrogen flows', Int. J. of Engineering Systems Modelling and Simulation.

Goncalves, E. and Zeidan, D. (2016b), Study of thermal effects in a cavitating venturi flow, in 'The 9th International Conference on Thermal Engineering Theory and Applications, March 24-26, 2016, Abu Dhabi, UAE'.

Huang, B., Wu, Q. and Wang, G. (2014), 'Numerical investigation of cavitating flow in liquid hydrogen', Int. Journal of Hydrogen Energy 39, 1698-1709.

Jameson, A., Schmidt, W. and Turkel, E. (1981), Numerical solution of the Euler equations by finite volume methods using Runge-Kutta time stepping schemes, in 'AIAA Paper 81-1259'.

Jones, W. and Launder, B. (1972), 'The prediction of laminarization with a two-equation model of turbulence', Int. J. Heat and Mass Transfer 15, 301-314.

Kapila, A., Menikoff, R., Bdzil, J., Son, S. and Stewart, D. (2001), 'Two-phase modeling of deflagration-to-detonation transition in granular materials: reduced equations', Physics of fluids 13(10), 3002-3024.

Kays, W. (1994), 'Turbulent Prandtl number - where are we?', ASME Journal of Heat Transfer 116, 284-295.

Liang, S., Liu, W. and Yuan, L. (2014), 'Solving seven-equation model for compressible two-phase flow using multiple GPUs', Computers $\mathcal{E}$ Fluids 99, 156-171. 
Menter, F. (1994), 'Two-equation eddy-viscosity turbulence models for engineering applications', AIAA Journal 32(8), 1598-1605.

Metayer, O. L., Massoni, J. and Saurel, R. (2004), 'Elaborating equations of state of a liquid and its vapor for two-phase flow models', Int. Journal of Thermal Sciences 43, 265-276.

Paniagua, G., Lavagnoli, S., Verstraete, T., Mahmoudi, W. and Benarama, T. (2013), 'Aero-design of transonic LH2 and LOx contra rotating turbopumps in an expander rocket engine', International Journal of Numerical Methods for Heat 85 Fluid Flow 23, $575-587$.

Petkovsek, M. and Dular, M. (2013), 'IR measurements of the thermodynamic effects in cavitating flow', International Journal of Heat and Fluid Flow 44, 756-763.

Prosperetti, A. and Plesset, M. (1978), 'Vapour-bubble growth in a superheated liquid', Journal of Fluid Mechanics 85, 349-368.

Rodio, M. and Abgrall, R. (2015), 'An innovative phase transition modeling for reproducing cavitation through a five-equation model and theoretical generalization to six and seven-equation models', Int. Journal of Heat and Mass Transfer 89, 1386-1401.

Roe, P. (1981), 'Approximate Riemann solvers, parameters vectors, and difference schemes', Journal of Computational Physics 43, 357-372.

Saurel, R. and Metayer, O. L. (2001), 'A multiphase model for compressible flows with interfaces, shocks, detonation waves and cavitation', Journal of Fluid Mechanics 431, 239-271.

Saurel, R., Petitpas, F. and Abgrall, R. (2008), 'Modelling phase transition in metastable liquids: application to cavitating and flashing flows', Journal of Fluid Mechanics 607, 313-350. 
Smith, B. (1994), A near wall model for the $k-l$ two equation turbulence model, in 'AIAA 94-2386, 25 ${ }^{\text {sh }}$ Fluid Dynamics Conference - Colorado Springs, Colorado'.

Spalart, P. and Allmaras, S. (1994), 'A one-equation turbulence model for aerodynamic flows', La Recherche Aérospatiale (1), 5-21.

Stahl, H., Stepanoff, A. and Phillipsburg, N. (1956), 'Thermodynamic aspects of cavitation in centrifugal pumps', Journal of Fluids Engineering 78, 1691-1693.

Tseng, C. and Shyy, W. (2010), 'Modeling for isothermal and cryogenic cavitation', Int. Journal of Heat and Mass Transfer 53, 513-525.

Utturkar, Y., Wu, J., Wang, G. and Shyy, W. (2005), 'Recent progress in modelling of cryogenic cavitation for liquid rocket propulsion', Progress in Aerospace Sciences 41, 558-608.

Voller, V. (2016), 'Computations of anomalous phase change', International Journal of Numerical Methods for Heat \& Fluid Flow 26, 624-638.

Wallis, G. (1967), 'One-dimensional two-phase flow', New York: McGraw-Hill.

Wang, J., Wang, Y., Liu, H., Huang, H. and Jiang, L. (2015), 'An improved turbulence model for predicting unsteady cavitating flows in centrifugal pump', International Journal of Numerical Methods for Heat \&3 Fluid Flow 25, 1198-1213.

Wilcox, D. (1988), 'Reassement of the scale-determining equation for advanced turbulence models', AIAA Journal 26(11), 1299-1310.

Zeidan, D. (2016), 'Assessment of mixture two-phase flow equations for volcanic flows using Godunov-type methods', Applied Mathematics and Computation 272, 707-719.

Zeidan, D., Touma, R. and Slaouti, A. (2014), 'Application of a thermodynamically compatible two-phase flow model to the high-resolution simulations of compressible gasmagma flow', International Journal for Numerical Methods in Fluids 76, 312-330. 


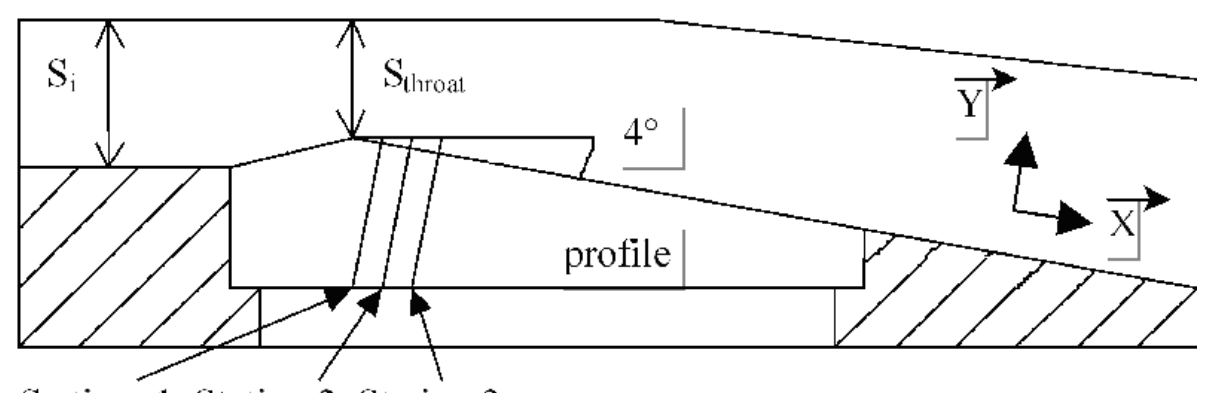

Station 1 Station 2 Station 3

Figure 1: Schematic view of the Venturi profile. 


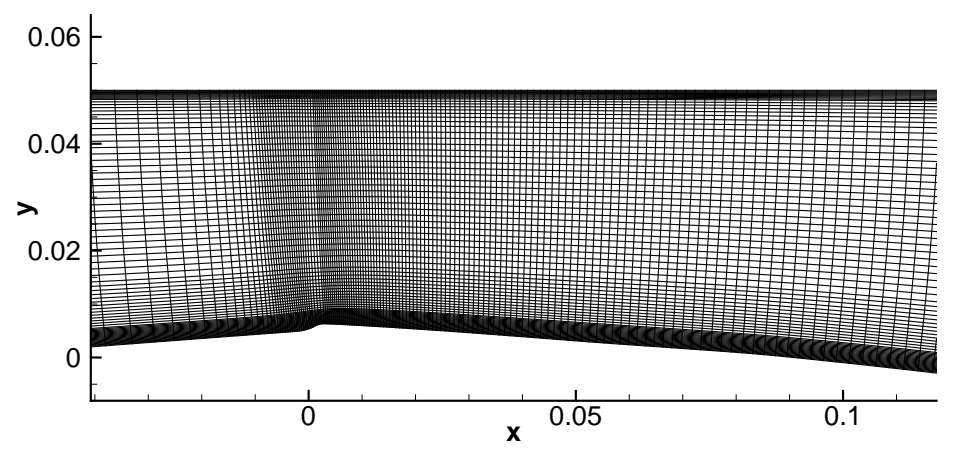

Figure 2: Enlargement of the mesh near the throat. 

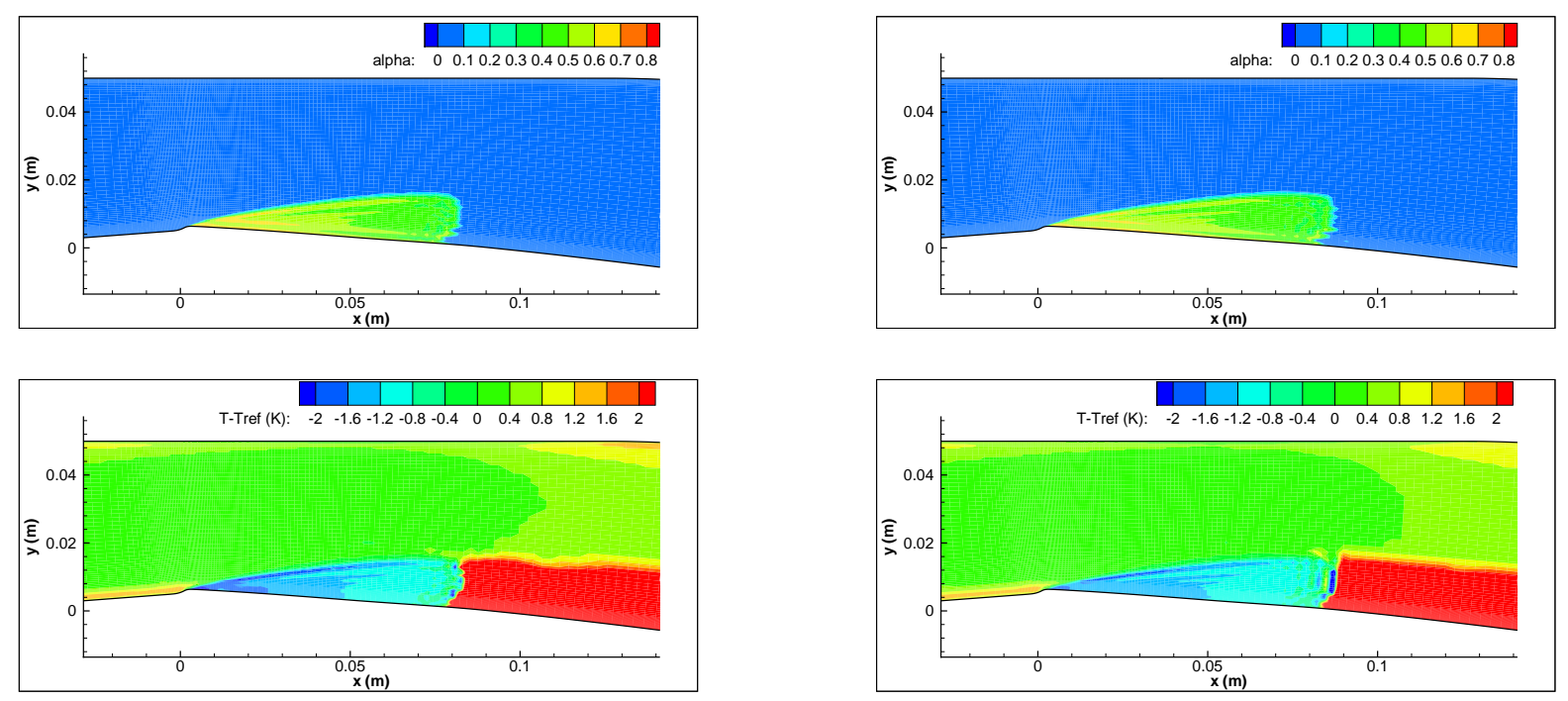

Figure 3: Void ratio and temperature difference $T-T_{\text {ref }}(\mathrm{K})$ contours, SST Menter model (left) and $k-\varepsilon$ Jones-Launder model (right). 

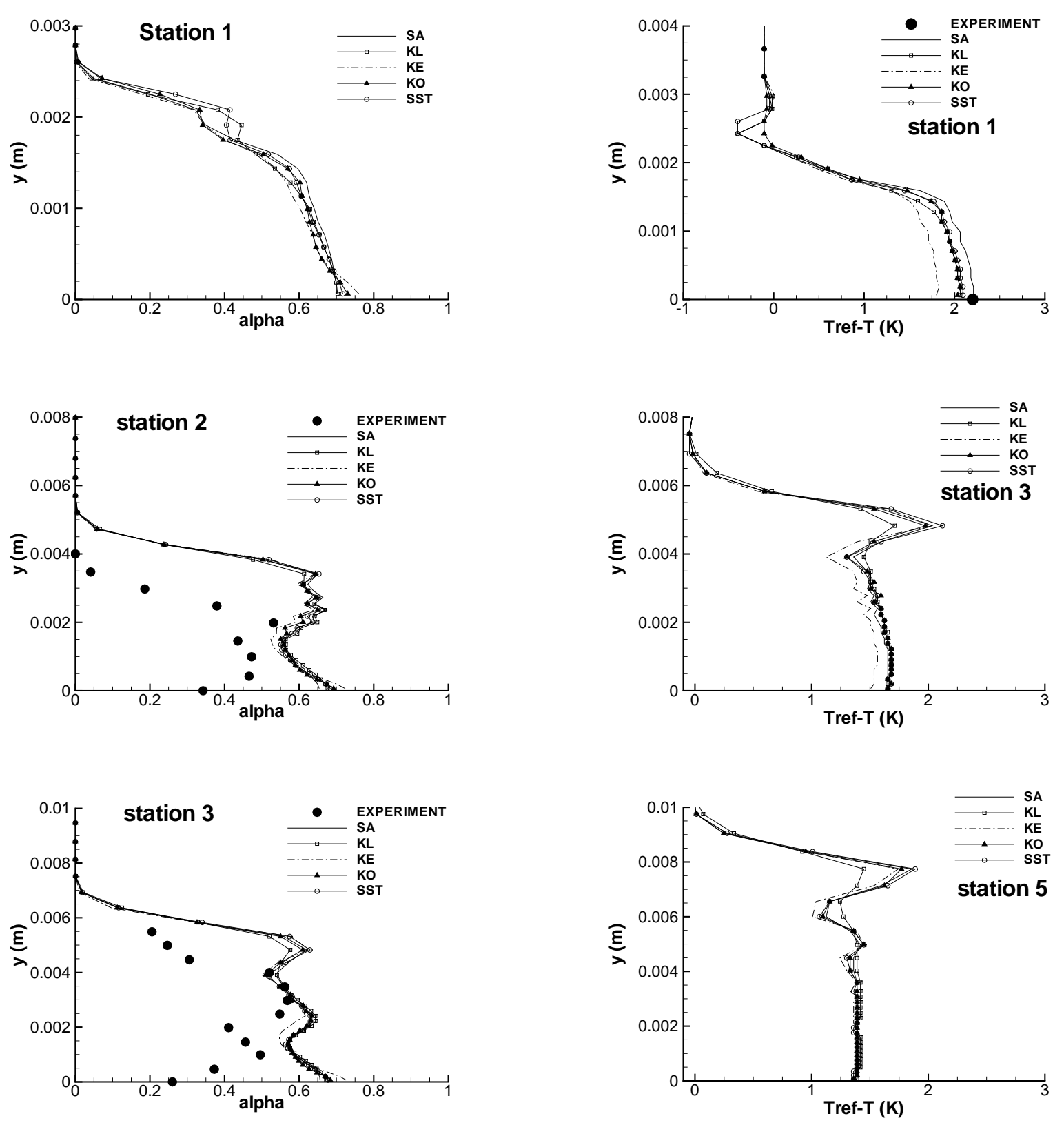

Figure 4: Profiles of void ratio and temperature drop $T_{r e f}-T$ at different stations, turbulence model influence. 

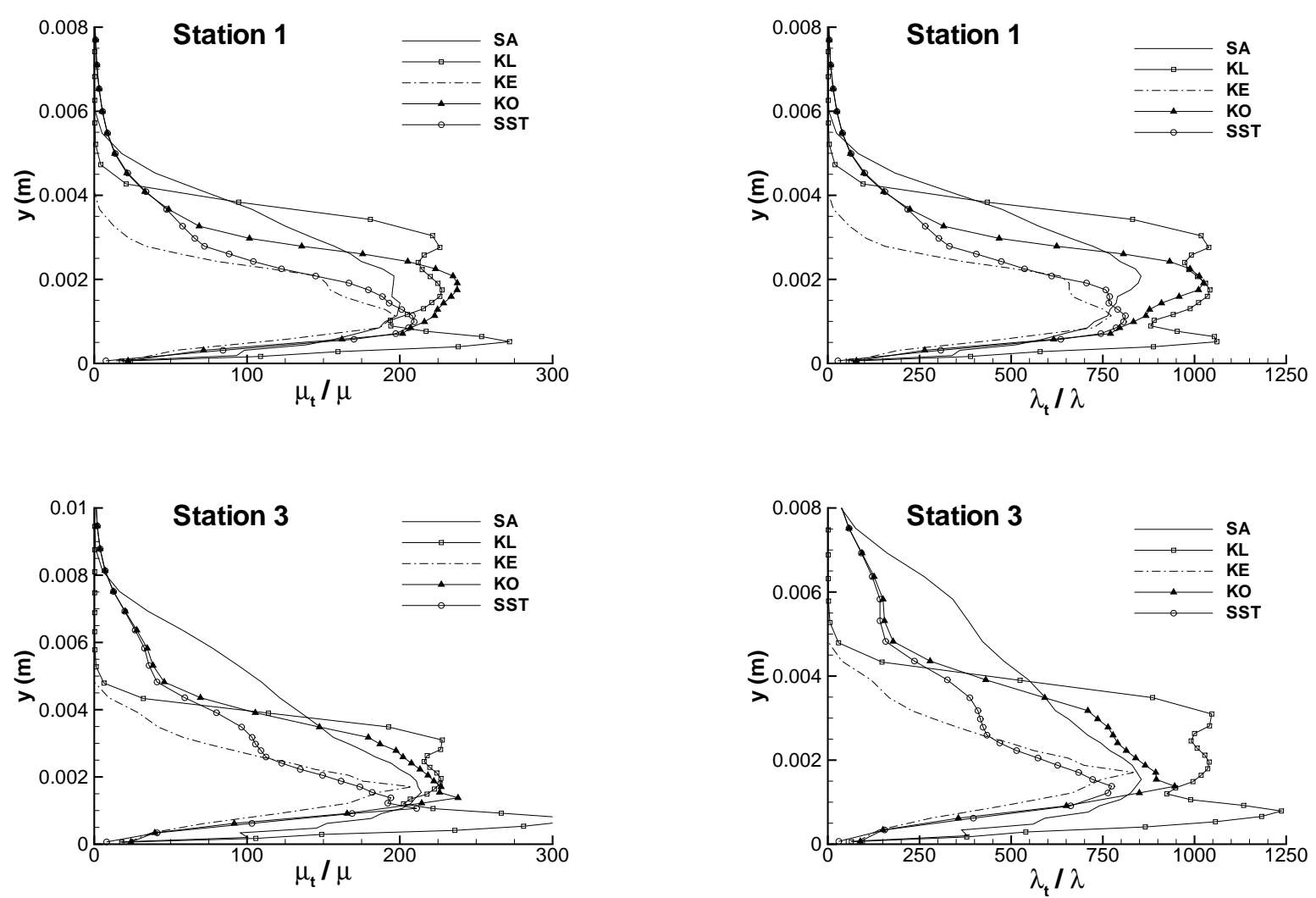

Figure 5: Profiles of ratios $\mu_{t} / \mu$ (left) and $\lambda_{t} / \lambda$ (right) at stations 1 and 3 , turbulence model influence. 

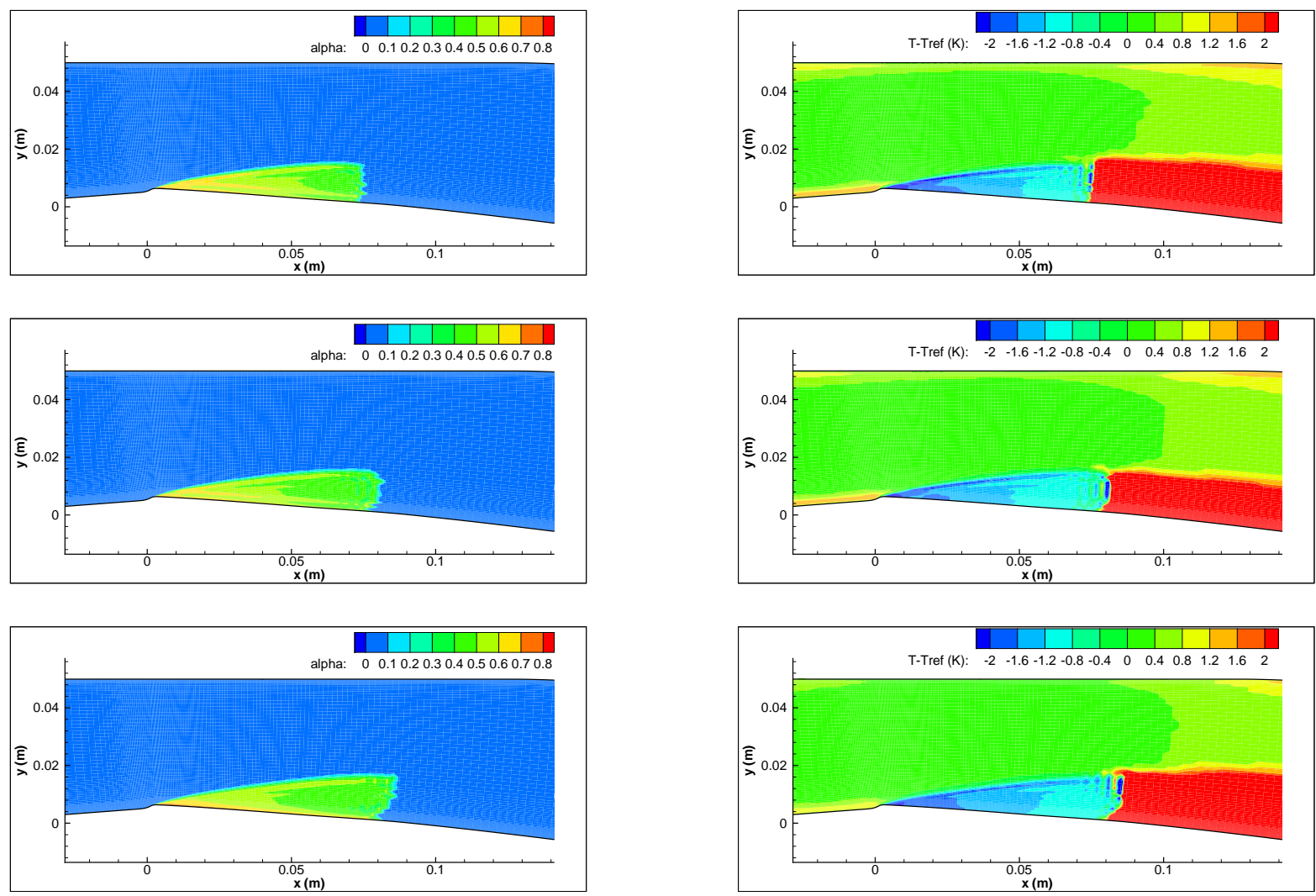

Figure 6: Void ratio (left) and temperature difference $T-T_{\text {ref }}(\mathrm{K})$ (right), turbulent Prandlt number influence, $P_{r_{t}}=1.5$ (top), $P_{r_{t}}=1$ (middle) and $P_{r_{t}}=0.25$ (down). 

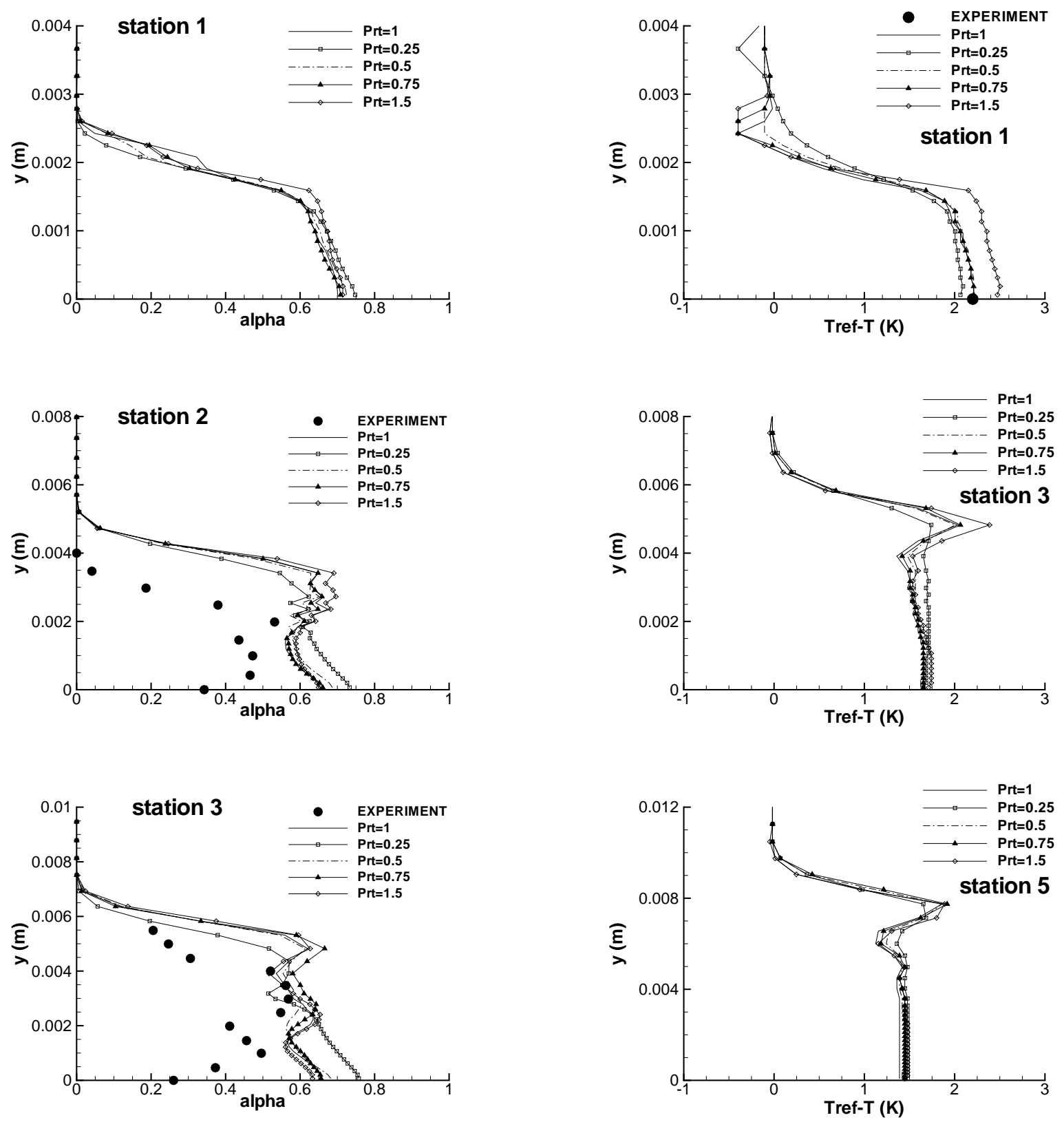

Figure 7: Profiles of void ratio and temperature drop $T_{r e f}-T$ at different stations, turbulent Prandlt number influence. 

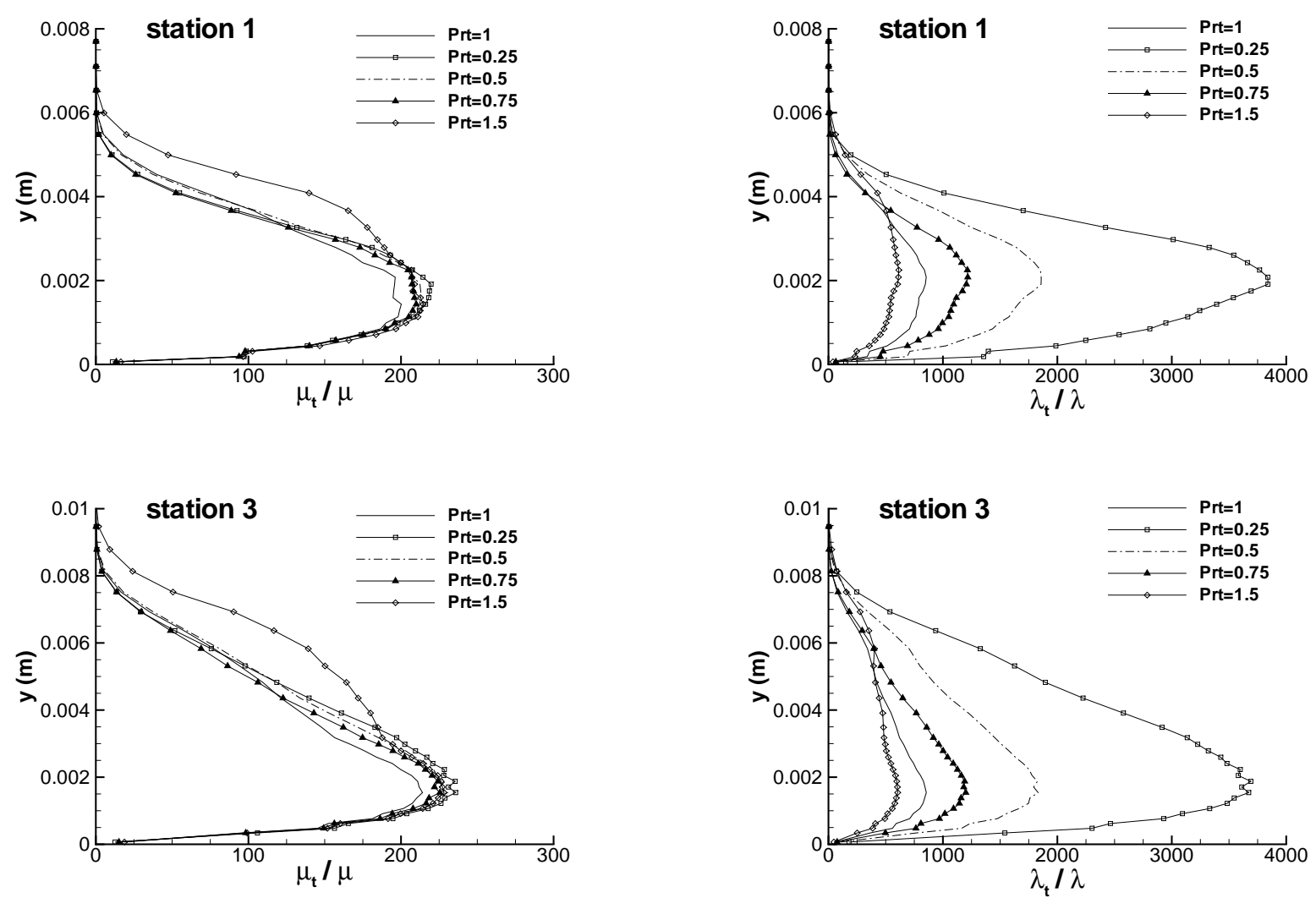

Figure 8: Profiles of ratios $\mu_{t} / \mu$ (left) and $\lambda_{t} / \lambda$ (right) at stations 1 and 3 , turbulent Prandlt number influence. 


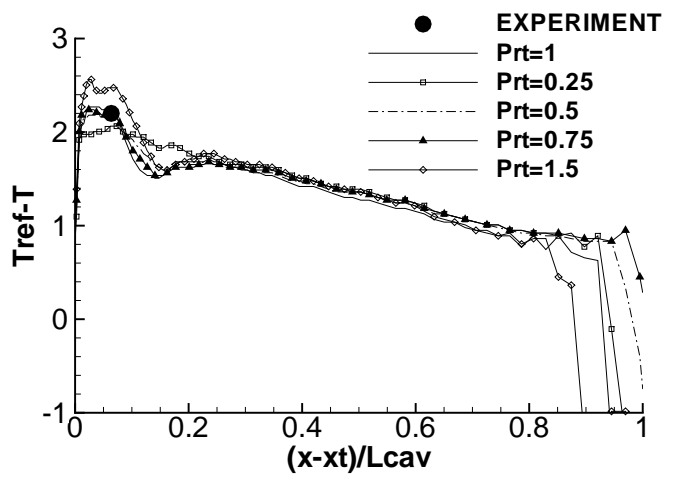

Figure 9: Wall temperature depression $T_{r e f}-T(\mathrm{~K})$ inside the divergent, turbulent Prandlt number influence. 